\title{
Detection of Molecular Adsorbate in Aqueous Solution Based on Electroosmosis
}

\author{
Toyohiro Naito, ${ }^{1 *}$ Hiroki Inoue, ${ }^{1}$ Shunta Futagami, ${ }^{2}$ \\ Akihiro Kunisawa, ${ }^{1}$ Takuya Kubo, ${ }^{1}$ and Koji Otsuka ${ }^{1}$ \\ ${ }^{1}$ Department of Material Chemistry, Graduate School of Engineering, Kyoto University, \\ Katsura, Nishikyo-ku, Kyoto 615-8510, Japan \\ ${ }^{2}$ Department of Chemical Engineering (CHIS), Vrije Universiteit Brussel, Pleinlaan 2, 1050 Brussels, Belgium
}

(Received June 30, 2018; accepted August 24, 2018)

Keywords: microfluidic device, electroosmotic flow, chemical sensor

We present a novel chemical sensor based on electroosmotic flow (EOF) for the simple detection of chemical adsorption in an aqueous solution. Fundamental investigations for the optimization of a microfabricated electroosmotic pump (EOP) revealed the relationship between the applied electric condition and pumping performance of EOPs. The optimized EOP had an improved minimum driving voltage of only $9 \mathrm{~V}$, which could be driven with a portable battery. The EOP after surface modification by molecular imprinting, such as a method with ionic complexes, changed the pumping performance by the adsorption of chemicals on its surface. We also investigated the relationship between the change in pumping rate and the chemical species of adsorbate using quaternary ammonium cations with different alkyl chains. The pump showed a change in pumping performance depending on the length of the alkyl chains.

\section{Introduction}

An electroosmotic pump (EOP) is a small pump based on electroosmotic flow (EOF), which was operated by a low voltage without pulsation and mechanical noise. For practical application, many types of EOP, such as polymer-based, ${ }^{(1-5)}$ inorganic, ${ }^{(6-9)}$ and microfabricated ${ }^{(10-18)}$ EOPs, have been developed so far. In particular, the microfabricated EOP has great potential as the micropump of point-of-care testing devices because microfluidic techniques can integrate various experimental operations on a small substrate.

The cause of EOF is the diffusion layer formed near the solid-liquid interface. ${ }^{(19,20)}$ In the case of a solid with a negatively charged surface, some cations bind strongly to the surface and partially neutralize the negative charge of the surface. The remaining negative charge attracts more cations, forming the diffusion layer. The diffusion layer neutralizes the net charge gradually to zero at the bulk liquid. Because cations in the diffusion layer interact loosely with the surface, they can be moved by Coulomb force under an electric potential along the liquid. This motion of the cations causes the bulk liquid near material surfaces to flow from the 
cathode to the anode. Porous materials and microfabricated devices generate EOF effectively owing to their high specific surface area and they are suitable materials for EOPs.

The velocity of EOF is proportional to the magnitude of electric field $(E)$ and also depends

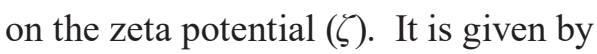

$$
v_{e o f}=\varepsilon \zeta E / \eta
$$

where $v_{e o f}$ is the velocity of EOF, $\varepsilon$ is the liquid permittivity, and $\eta$ is the viscosity of the liquid. When some ionic compounds are mixed in the liquid and adsorb on the material surface, the velocity of EOF should be changed by varying the zeta potential (Fig. 1). The change in EOF velocity can be applied to adsorbate sensing.

In this study, we describe a novel method of chemical substance detection in an aqueous solution by evaluating the change in the pumping rate of the EOP. The EOP can integrate two crucial functions for a portable analysis system, i.e., a pump and a sensor. The effects of microstructures and surface functional groups on pump performance are investigated to improve the basic pumping performance. The concept of chemical sensing is demonstrated after the optimization of the microfabricated EOP.

\section{Materials and Methods}

\subsection{Device design and fabrication}

The microfabricated EOP had a microstructure array region that divided a wide microfluidic channel into several narrow channels to increase surface area [Fig. 2(a)]. The width of narrower channels was $10 \mu \mathrm{m}$ and the length and number of narrower channels were changed to investigate structural effects on pump performance. The width of the wide microfluidic channel increased from 1510 up to $3010 \mu \mathrm{m}$ with increasing the number of narrower channels.

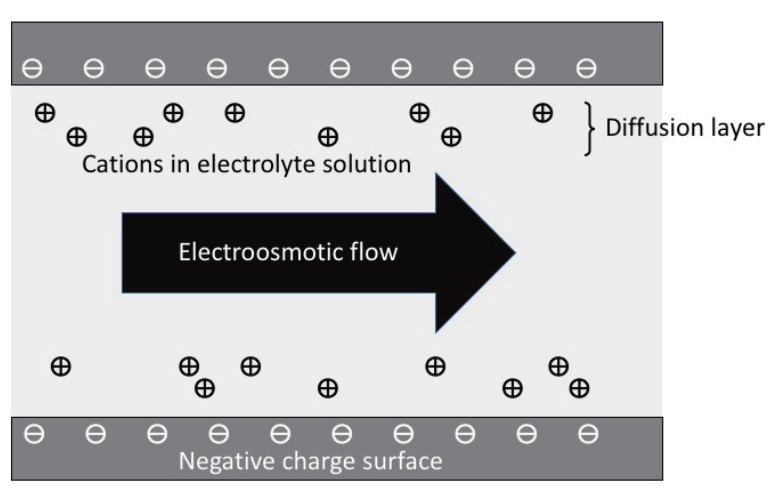

(a)

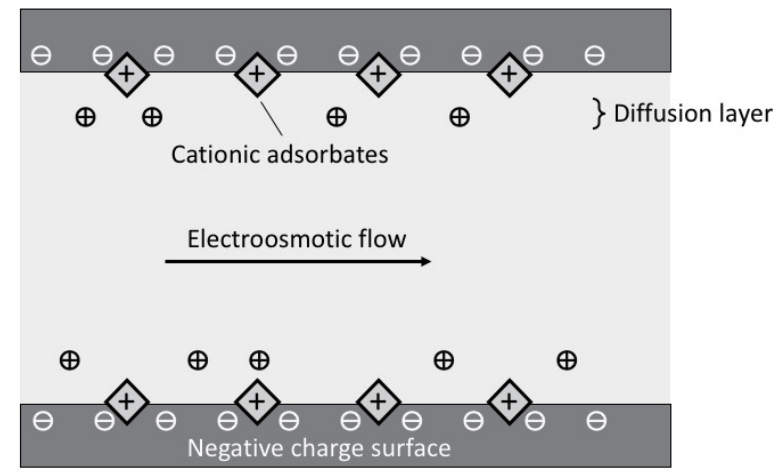

(b)

Fig. 1. Schematic illustrations of the working principle of molecular detection. EOF (a) before and (b) after adsorption. 


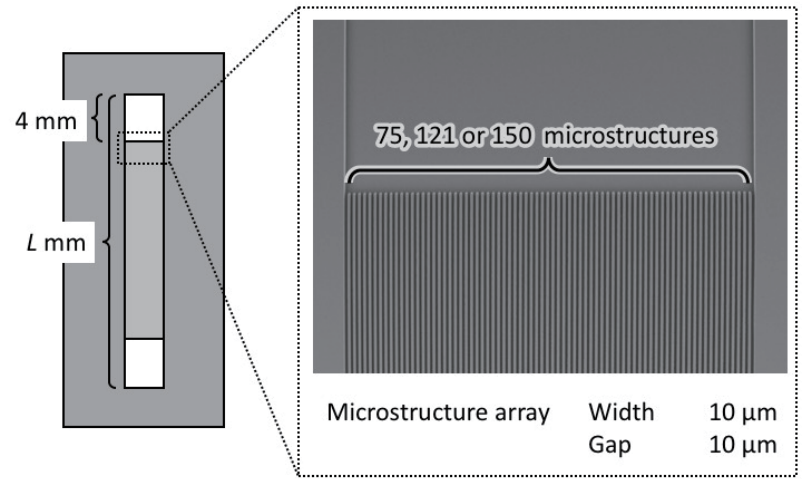

(a)

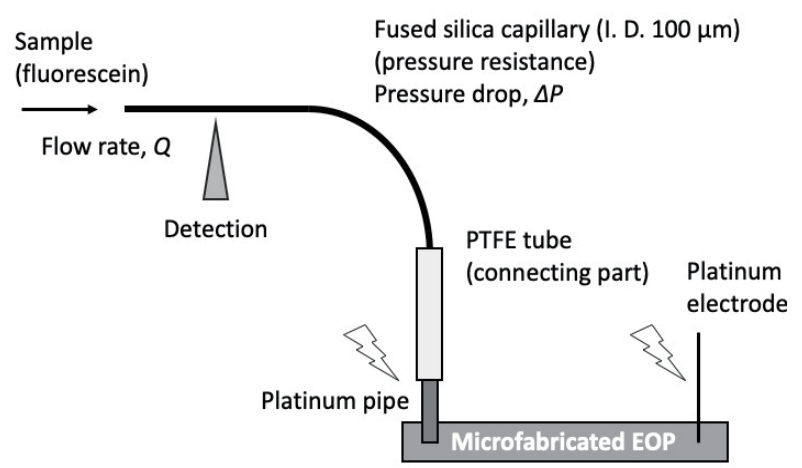

(b)

Fig. 2. Schematic illustrations of (a) the microfluidic EOP and (b) the measurement system for the evaluation of pumping function.

Microfluidic devices were made from poly(dimethylsiloxane) (PDMS) by conventional soft lithography. A photoresist (SU-8 3050, MicroChem Corp.) was used to form a mold for casting the PDMS devices by photolithography. The photoresist was diluted with cyclopentanone to reduce viscosity to create a 15- $\mu$ m-thick mold. A two-component kit (Sylgard 184, Dow Corning Toray Co., Ltd.), including a prepolymer of PDMS and a curing agent, was used for PDMS fabrication; the two components were mixed in a 10:1 weight ratio. The resulting mixture was thoroughly degassed in vacuum, poured into the mold, and degassed in vacuum again and cured for $30 \mathrm{~min}$ at $100{ }^{\circ} \mathrm{C}$. The PDMS and a glass slide were bonded covalently after 1 min of plasma treatment.

\subsection{Evaluation of pump performance}

A schematic diagram of the measurement system is shown in Fig. 2(b). The PDMS devices are connected to a fused silica capillary with a platinum pipe and a poly(tetrafluoroethylene) (PTFE) tube. The fused silica capillary works as a resistance tube and reduces the pressure generated from the microfluidic device. The other end of the fused silica capillary has a detection window and is connected to a sample reservoir.

The fused silica capillary is filled with buffer or deionized water. Once electric field is applied to a microfluidic EOP, $200 \mu \mathrm{M}$ fluorescein diluted with methanol at the sample reservoir is introduced into the capillary. The fluorescence intensity variation is measured with a fluorescence microscope at the detection window to calculate the pumping rate. The fluorescence intensity increases gradually and finally reaches a steady value. The maximal value of the first derivative of fluorescence intensity variation is defined as the detection time of fluorescein. The pumping rate is obtained by dividing the capillary volume from the sample reservoir to the detection window by the detection time. The length of the capillary is changed to control the pressure drop $(\Delta P)$. The pumping rate is plotted against the pressure drop. The pressure drop in the fused silica capillary and PTFE tube calculated using Eq. (2) is the sum of Hagen-Poiseuille equations for the capillary and PTFE tube. 


$$
\Delta P=128 \eta Q l /\left(\pi d^{4}\right)
$$

Here, $\Delta P$ is the pressure drop at the capillary, $Q$ is the pumping rate, $l$ is the length of the capillary, and $d$ is the diameter of the capillary. The $x$ - and $y$-intercepts of the $\Delta P-Q$ plot indicate the intrinsic pumping pressure and maximum pumping rate of the microfluidic EOP, respectively.

\subsection{Surface modification}

Microfluidic channel surfaces were modified with various functional groups. A microfluidic EOP was flushed with $1 \mathrm{M} \mathrm{HCl}$ aq. for $10 \mathrm{~min}$ and deionized water for $5 \mathrm{~min}$. It was filled with $10 \%(\mathrm{v} / \mathrm{v}) 3$-aminopropyltriethoxysilane (APTES)/ethanol for $1 \mathrm{~min}$ at room temperature to introduce amino groups on the surfaces. A $20 \mathrm{mM}$ ionic complex composed of benzyltributylammonium chloride (BTBA) and 4-sulfobenzoic acid in methanol was reacted with 4-(4,6-dimethoxy-1,3,5-triazin-2-yl)-4-methylmorpholinium chloride (DMT-MM) for $48 \mathrm{~h}$ at room temperature. The ionic complex was obtained by mixing BTBA $(2.0 \mathrm{~g})$ and 4-sulfobenzoic acid potassium salt $(1.7 \mathrm{~g})$ in water $(50 \mathrm{~mL})$ in our previous study. ${ }^{(21)}$ After the reaction of the ionic complex and amino groups on the surface, BTBA was removed by flushing a $2 \mathrm{M} \mathrm{NaCl}$ aqueous solution overnight at room temperature.

\subsection{Adsorption test}

Ammonium chloride (AC), tetraethylammonium chloride (TEAC), and tetrabutylammonium chloride (TBAC) were used as adsorbates. An optimized microfluidic pump has a $6.0 \mathrm{~mm}$ length with a 4.0-mm-long microstructure array region, a $3010 \mu \mathrm{m}$ width, and a $15 \mu \mathrm{m}$ depth. The microstructures were $10 \mu \mathrm{m}$ in width and were arrayed with $10 \mu \mathrm{m}$ gap distances. The number of arrayed microstructures in the microfluidic pump was 150 .

Each $10 \mathrm{mM}$ adsorbate aqueous solution was filled into the optimized microfluidic pump for $1 \mathrm{~min}$ to interact with the adsorbate and surfaces. Free adsorbate molecules in the microfluidic pump were flushed with water. After the absorption of molecules, the pump performance was characterized with a 54-mm-long fused silica capillary (I.D. $100 \mu \mathrm{m}$ ) and an applied voltage of $100 \mathrm{~V}$.

\section{Results and Discussion}

\subsection{Evaluation of structural effects of microstructures on pump performance}

Figure 3(a) shows $\Delta P-Q$ plots for a 30-mm-long pump and a 60 -mm-long pump. The shorter pump generates a twofold higher pumping rate than the longer pump at the same applied voltage. This is because the EOF velocity is proportional to the electric field strength according to the Helmholtz-Smoluchowski equation, and the electric field strength in the shorter pump is two times higher than that in the longer pump. 


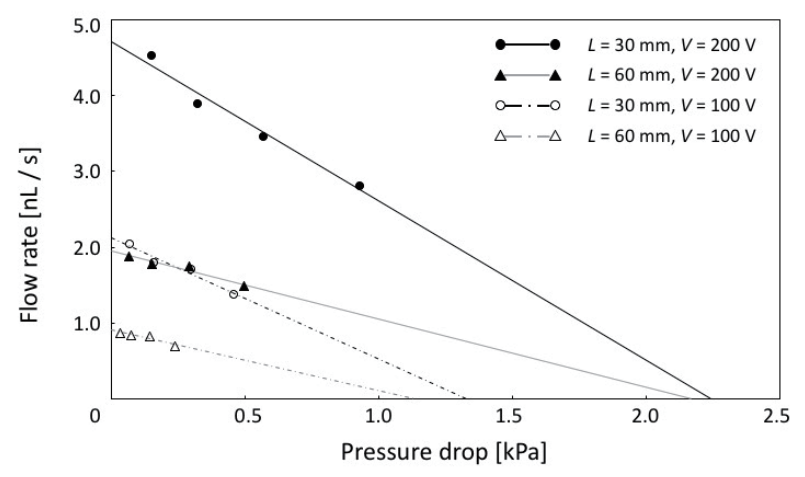

(a)

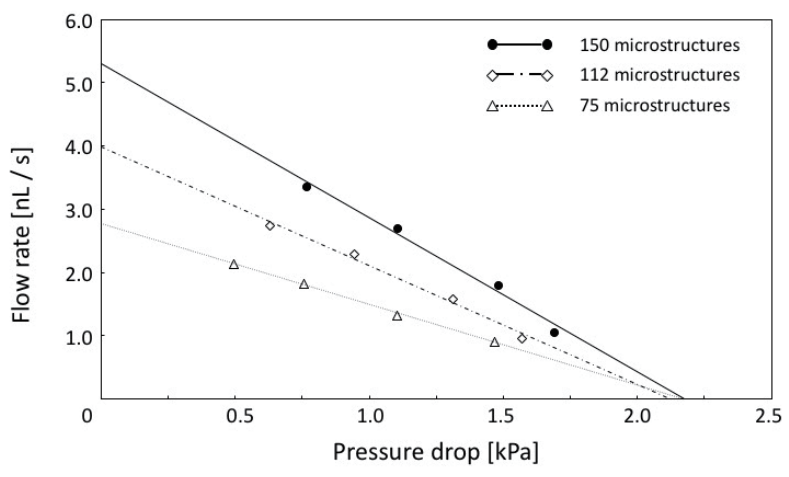

(b)

Fig. 3. $\quad P-Q$ plots of microfluidic EOPs. (a) Dependence on electric field strength and (b) dependence on number of microstructures.

The noteworthy point is that two pumps of different lengths indicate approximately the same maximum pressure at the same applied voltage. The maximum pumping pressures generated under different strengths of the electric field were proportional to the electric field strength. Substituting Eq. (2) into Eq. (1) and rearranging yield, we obtain

$$
\Delta P=32 \varepsilon \zeta E l / d^{2}
$$

The pressure drop is the difference between the pumping pressure $(P)$ and the pressure at the end with the sample reservoir $\left(P_{i n}\right)$ in this case. The substitution of this relation into Eq. (3) gives

$$
P=32 \varepsilon \zeta E l / d^{2}+P_{i n}
$$

The $x$-intercept of the $P-Q$ plot gives the maximum pumping pressure $\left(P_{\max }\right)$ that is proportional to the electric field strength, because $P_{i n}$ is equal to 0 at that point. By contrast, connecting pumps in series improves the pumping pressure and the total pumping pressure is the sum of those of each pump. If a long pump is regarded as a series of shorter pumps, the maximum pressure should have a linear relationship with the pump length $(L)$.

$$
P_{\max }=32 \varepsilon \zeta E L l / d^{2}=32 \varepsilon \zeta V l / d^{2}
$$

That is why the maximum pumping pressure is proportional to the applied voltage $(V)$ between the two ends of the microfluidic pump.

Figure 3(b) shows the performances of microfluidic pumps with different numbers of microstructures. Although the maximum pumping pressure was independent of the number of microstructures, the maximum pumping rate was proportional to the number of microstructures. The increase in the number of microstructures increases the cross-sectional area in the microfluidic pump. A larger cross-sectional area generates a higher pumping rate at the same EOF velocity, which is determined by the applied electric field. 
According to the above results, the high electric field strength and a large cross-sectional area in the microfluidic pump increase the pumping rate. This means that the optimum microfluidic EOP is short to enhance the electric field strength and a large number of microstructures to provide a large cross-sectional area. The optimized microfluidic pump generates 6.6 times higher pumping rates. It was driven at only $9 \mathrm{~V}$ and generated $1.6 \pm 0.1 \mathrm{~nL}$ against the $54 \mathrm{~mm}$ fused silica capillary.

\subsection{Evaluation of chemical effects of material surface on pump performance}

The EOF velocity of the microfluidic pumps after modification with APTES was measured in order to evaluate the surface chemical condition. The microfluidic pump modified with APTES generated the EOF from the cathode to the anode at $\mathrm{pH} 4.0$. This EOF was in the opposite direction to that caused by the silanol groups. Furthermore, few silanol groups are dissociated at $\mathrm{pH}$ 4.0. This EOF suggests that the surface modification with APTES was achieved to introduce amino groups on the internal surface of microfluidic EOPs.

Figure 4 shows the $P-Q$ plots of microfluidic pumps modified with APTES of different concentrations. The pump performance was increased by modifying the surface with a high concentration of APTES. The surface has more amino groups owing to effective modification with the high-APTES-concentration solution. The pumps modified with 10 and 100\% APTES solutions gave approximately the same performance. The number of amino groups on the modified surface reached the saturation point. Although we cannot quantify the number of amino groups on the modified surface, this result suggests that the pump performance is dependent on the number of ionic functional groups.

\subsection{Detection of adsorbates on microfabricated structure surfaces}

For the proof of concept, we evaluated the pumping performance of the sulfo-modified EOP against the $54 \mathrm{~mm}$ resistance fused silica capillary. The sulfo-modified EOP without interaction with BTBA generated a pumping rate of $4.58 \mathrm{~nL} / \mathrm{s}$. The pumping rate was reduced to 3.81 $\mathrm{nL} / \mathrm{s}$ after adsorbing BTBA. The sulfo group was successfully introduced on the surface of the

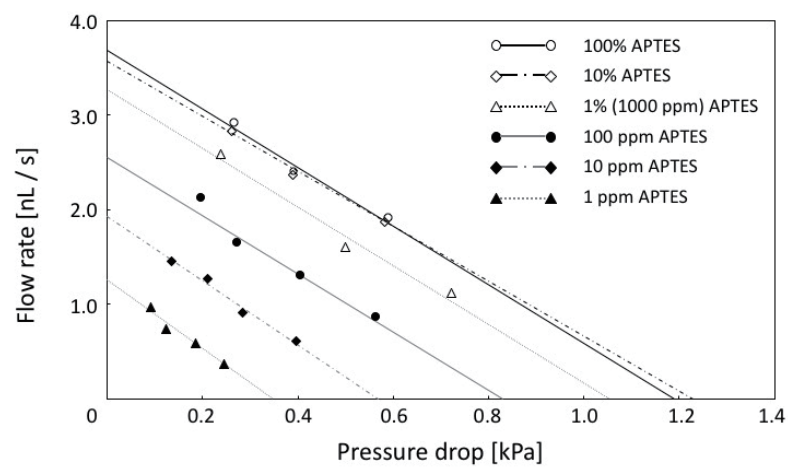

Fig. 4. $\quad P-Q$ plots of microfluidic EOPs modified with different concentrations of APTES. 


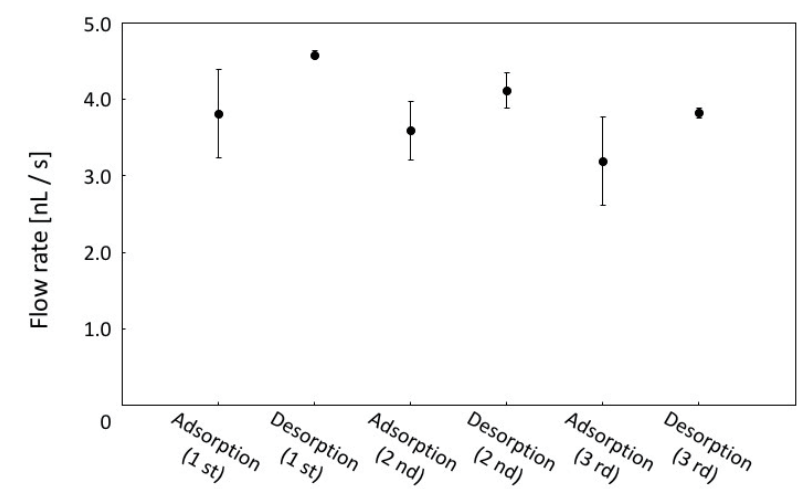

Fig. 5. Flow rates of microfluidic pumps with BTBA adsorption and without BTBA on its surface.

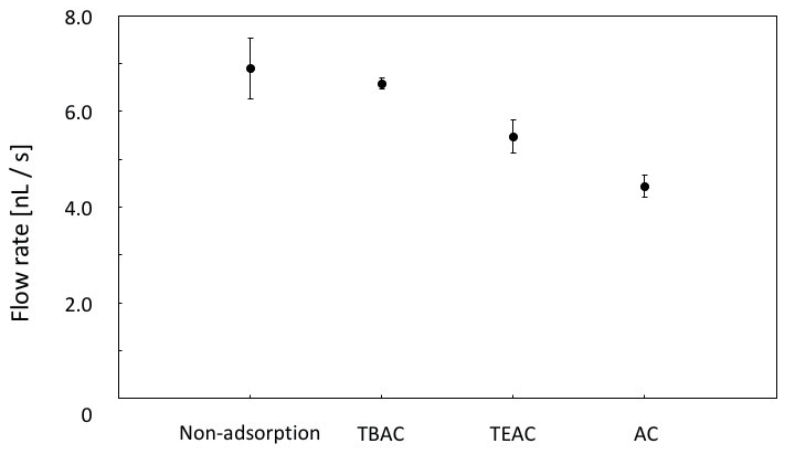

Fig. 6. Flow rates of microfluidic pumps with adsorption of different chemical species.

EOP and it interacted with the cationic sample, BTBA. However, water in the EOP flows from the cathode to the anode, which is the opposite direction of what we expected. The reaction between the ionic complex and the amino group on the surface was inadequate and the amino group remained on the surface. The EOF from the cathode to the anode was caused mainly by the amino group and suppressed by the sulfo group. When BTBA was adsorbed on the surface, the electric charge derived from the sulfo group was cancelled by the ionic interaction with BTBA and some amino groups were covered by the alkyl chain of BTBA. This interaction between the sulfo group and BTBA reduces the surface positive charge. The pumping rate of the microfluidic pump decreased whenever BTBA adsorbed on the surface (Fig. 5). Although the surface modification and the material of the microfluidic EOP should be improved to realize more sensitive sensing, the microfluidic pump worked as an adsorbate sensor.

\subsection{Dependence of change in pumping rate on chemical species of adsorbates}

The relationship between the change in the pumping rate and adsorbing chemical species was investigated using quaternary ammonium cations with different alkyl chains as the adsorbate (Fig. 6). We predicted that the EOP with TBAC would show the lowest pumping rate, because the longer alkyl chains of the adsorbate have the potential to cover more amino groups on the surface. However, AC and TEAC reduce the pumping rate, and the EOP with AC adsorption shows the lowest pumping rate. This result indicates that steric effects or electron donation from adsorbates with a long alkyl chain hinders the interaction between adsorbates and amino groups.

\section{Conclusions}

In this study, we adopted a microfluidic EOP as a chemical sensor. A short microfluidic channel with an array of a large number of microstructures could generate a high pumping rate. The optimum microfluidic EOP with sulfo-modified surfaces interacted with ammonium 
cations. The adsorption of ammonium cations could be detected by evaluating the change in pumping performance. The pumping performance was also changed by the chemical species of the adsorbate. Since the optimum microfluidic EOP could be driven by only $9 \mathrm{~V}$, it can be used as a portable device. We expect that a combination of molecular imprinting technologies will realize the development of the new type of molecule-selective sensor.

\section{Acknowledgments}

This work was partly supported by the Micro/Nano Fabrication Hub in Kyoto University founded by the Ministry of Education, Culture, Sports, Science and Technology (MEXT), Japan. We would also like to express our gratitude to the Asahi Glass Foundation, Shimadzu Foundation, and the Murata Science Foundation for their financial support.

\section{References}

1 K. Bengtsson, J. Christoffersson, C.-F. Mandenius, and N. D. Robinson: Microfluid. Nanofluid. $22-27$ (2018) 27.

2 L. Zhou, J. J. Lu, C. Gu, and S. Liu: Anal. Chem. 86 (2014) 12214. https://doi.org/10.1021/ac503223r

3 C. Gu, Z. Jia, Z. Zhu, C. He, W. Wang, A. Morgan, J. J. Lu, and S. Liu: Anal. Chem. 84 (2012) 9609. https:// doi.org/10.1021/ac3025703

4 W. Wang, C. Gu, K. B. Lynch, J. J. Lu, Z. Zhang, Q. Pu, and S. Liu: Anal. Chem. 86 (2014) 1958. https://doi. org/10.1021/ac4040345

5 A. Chen, K. B. Lynch, J. Ren, Z. Jia, Y. Yang, J. J. Lu, and S. Liu: Anal. Chem. 89 (2017) 10806. https://doi. org/10.1021/acs.analchem.7b02132

6 S. Zeng, C. H. Chen, J. C. Mikkelsen, Jr, and J. G. Santiago: Sens. Actuators, B 79 (2001) 107. https://doi. org/10.1016/S0925-4005(01)00855-3

7 A. Gupta, H. Denver, A. H. Hirsa, J. A. Stenken, and D. A. Borca-Tasciuc: Appl. Phys. Lett. 91 (2007) 094101. https://doi.org/10.1063/1.2775836

8 Z. Cao, L. Yuan, Y. F. Liu, S. Yao, and L. Yobas: Microfluid. Nanofluid. 13 (2012) 279. https://doi.org/10.1007/ s10404-012-0959-x

9 N. Lewpiriyawong, G. Xu, and C. Yang: Electrophoresis 39 (2018) 878. https://doi.org/10.1002/elps.201700395

10 H. Salimi-Moosavi, T. Tang, and D. J. Harrison: J. Am. Chem. Soc. 119 (1997) 8716. https://doi.org/10.1021/ ja971735f

11 R. B. Schasfoort, S. Schlautmann, J. Hendrikse, and A. van den Berg: Science. 286 (1999) 942. https://doi. org/10.1126/science.286.5441.942

12 M. E. Piyasena, R. Newby, T. J. Miller, B. Shapiro, and E. Smela: Sens. Actuators, B 141 (2009) 263. https:// doi.org/10.1016/j.snb.2009.05.014

13 I. M. Lazar and B. L. Karger: Anal. Chem. 74 (2002) 6259. https://doi.org/10.1021/ac0203950

14 Y. Takamura, H. Onoda, H. Inokuchi, S. Adachi, A. Oki, and Y. Horiike: Electrophoresis 24 (2003) 185. https://doi.org/10.1002/elps.200390012

15 B. P. Cahill, L. J. Heyderman, J. Gobrecht, and A. Stemmer: Phys. Rev. E: Stat. Nonlinear Soft Matter Phys. 70 (2004) 036305. PMID:15524631. https://doi.org/10.1103/PhysRevE.70.036305

16 K. Seibel, L. Schöler, H. Schäfer, and M. Böhm: J. Micromech. Microeng. 18 (2008) 025008. https://doi. org/10.1088/0960-1317/18/2/025008

17 X. Wu, P. Ramiah Rajasekaran, and C. R. Martin: ACS Nano 10 (2016) 4637. https://doi.org/10.1021/ acsnano.6b00939

18 H. V. Funetes and A. T. Woolley: Lab Chip 7 (2007) 1524. https://doi.org/10.1039/b708865e

19 M. R. Hossan, D. Dutta, N. Islam, and P. Dutta: Electrophoresis 39 (2018) 702. https://doi.org/10.1002/ elps. 201700375

20 L. Chen, J. Ma, and Y. Guan: J. Chromatogr. A 1028 (2004) 219. https://doi.org/10.1016/j.chroma.2003.11.071

21 T. Kubo, K. Kuroda, Y. Tominaga, T. Naito, K. Sueyoshi, K. Hosoya, and K. Otsuka: J. Pharm. Biomed. Anal. 89 (2014) 111. https://doi.org/10.1016/j.jpba.2013.10.040 